\title{
Near-UV nebular absorption lines of $\eta$ Carinae
}

\author{
Theodore R. Gull, Gladys L. Vieira, and Anthony C. Danks \\ NASA's Goddard Space Flight Center, \\ Code 681, Greenbelt, MD 20771, USA
}

\begin{abstract}
The notorious $\eta$ Carinae was observed with the STIS from $2380 \AA$ to $3160 \AA$ with a resolving power of $\sim 130000$. Over 500 absorption lines have been identified as originating from Fe I, Fe II, Ti II, V II, Cr II, Ni II, Co II, Mn II, MgI and Mg II. Most of the lines have multiple components ranging from $-145 \mathrm{~km} \mathrm{~s}^{-1},-385 \mathrm{~km} \mathrm{~s}^{-1}$ to $-587 \mathrm{~km} \mathrm{~s}^{-1}$. Many interstellar absorption velocity systems, also found in the spectra of other Carina stars, are seen in the spectrum of $\eta$ Car. While ISM absorption lines originate from the ground energy level, the nebular lines originate from energy levels well above the ground state. We are still evaluating the origin and location of these velocity systems in line of sight.
\end{abstract}

\section{Introduction}

The ultraviolet spectra of $\eta$ Carinae is extraordinarily complex in nature. Previous spectral line identifications were provided by Viotti et al. (1989) for IUE spectra recorded with $R=10000$ resolving power and a $10^{\prime \prime} \times 20^{\prime \prime}$ aperture. Depending upon position angle, most or all of the visible Homunculus was included within the aperture. Spectroscopic observations have been done with HST-STIS with $R=7000$ from $10300 \AA$ to $1640 \AA$ and $0{ }^{\prime} 1$ angular resolution (Gull et al. 2001). STIS, with resolving power $R=30000$ and 0 ' 05 angular resolution (Davidson, HST 8327, 9083), recorded spectra from $1175 \AA$ to $2350 \AA$. STIS resolves $\eta$ Car from the nebula. Indeed overlap spectra between FUSE and STIS in the $1175-1185 \AA$ region demonstrate that $95 \%$ of the far-UV is scattered by surrounding ejecta (Iping et al. 2001). The stellar spectrum below $2350 \AA$ proves to be very complex due to a superimposed forest of narrow line absorptions. The large apertures of FUSE and IUE smear the spectrum due to the nebular scattered light. Moreover, the Ba $\alpha$ P-Cygni line profiles, seen scattered off the Homunculus, change considerably with nebular position. Smith et al. (in preparation) interpret changes in the Balmer profiles as being due to the stellar wind being very different from the equatorial to the polar regions.

\section{The observations and interpretation}

The FUSE and the HST-STIS medium-resolution echelle data from $912-2350 \AA$ prove to be very complex with many narrow absorption lines. In an attempt to resolve these absorptions, we obtained STIS observations in the high-dispersion echelle mode in the near-UV. The stellar spectrum changes less rapidly than in the far-UV, the interstellar absorption spectrum - known through comparison with nearby stars in the Carina complex - is very dull. However, we found a 
multitude of narrow absorption lines! Most do not correlate with classical ISM absorption lines. Only when we plotted known ISM line profiles, such as Mg I, $\mathrm{Mg}$ II, and normally weak ISM lines, did we realize that there were many distinct velocity components. Twenty nebular velocity systems were identified: one at $-145 \mathrm{~km} \mathrm{~s}^{-1}$, and rest between $-385 \mathrm{~km} \mathrm{~s}^{-1}$ to $-587 \mathrm{~km} \mathrm{~s}^{-1}$. The $-145 \mathrm{~km} \mathrm{~s}^{-1}$ system has an intrinsic line width of about $10 \mathrm{~km} \mathrm{~s}^{-1}$; the rest have line widths of a few $\mathrm{km} \mathrm{s}^{-1}$. Then we found absorption lines arising from lower energy levels well elevated about the ground. For the $-145 \mathrm{~km} \mathrm{~s}^{-1}$ system, some of these lower levels appear to be as high as $4 \mathrm{eV}$ ! A high velocity system at $-513 \mathrm{~km} \mathrm{~s}^{-1}$ has many very narrow absorption lines with intrinsic widths of $2.3 \mathrm{~km} \mathrm{~s}^{-1}$ and with lower levels ranging from 0 to $0.1 \mathrm{eV}$. We found the following ions and atoms: $-147 \mathrm{~km} \mathrm{~s}^{-1}$ : FeII, CrII, Ni II, MnII, CoII and MgII; $-513 \mathrm{~km} \mathrm{~s}^{-1}$ : FeI, FeII, Ti II, Si I, Mg II, Mn II, V II, Ni I and Cr II. The presence of absorption lines from excited levels clearly indicates dense material close to the star!

$\eta$ Car has a very strong stellar wind with terminal velocity $v_{\infty}=550 \mathrm{~km} \mathrm{~s}^{-1}$. Likewise, much of the Homunculus outer structures, at $2.3 \mathrm{kpc}$ distance, is consistent with a large shell ejected in the late 1840's (Morse et al. 2001). Weis, Duschl \& Chu (1999) found long, nearly linear structures beyond the Homunculus with velocities as high as a few thousand $\mathrm{km} \mathrm{s}^{-1}$. The Homunculus is very lumpy in character. Polarization measurements by King et al. (2002) indicate the shell thickness is thin, lending to single scattering events.

The $-145 \mathrm{~km} \mathrm{~s}^{-1}$ absorption system, with its $10 \mathrm{~km} \mathrm{~s}^{-1}$ width and with lower ionic energy levels as high as $46000 \mathrm{~cm}^{-1}$, is located close to $\eta$ Car. The higher velocity systems are less easily placed. Their velocities are below the stellar wind terminal velocity, but the line widths and lower energy levels indicate that these systems must originate much further from the star. Likely, they exist within the Homunculus. However, a high dispersion spectrum of the blob Weigelt-D, 0!2 away from $\eta$ Car, does not exhibit strong absorption systems. The presence of vanadium, which has never been seen in the ISM, and other species are rarely seen. Grain modification, or the lack of dust grains, is a strong possibility. Finally, we note that column densities derived from lines originating from the same lower levels do not appear to agree. This may indicate errors in $g f$-values, but could also indicate that the absorbing systems do not fully cover the star, e.g., these may be small clumps with dimensions less that the stellar source. If true, we can anticipate dramatic variability in absorption with time.

Acknowledgments. This research was supported by STIS GTO resources under the HST Project. Observations were scheduled through STScI.

\section{References}

Gull, T.R., Ishibashi, K., Davidson, K., Collins, N. 2001, in: T.R. Gull, S. Johansson \& K. Davidson (eds.), Eta Carinae and Other Mysterious Stars, ASP-CS 242, 391

Iping, R.C., Sonneborn, G., Gull, T.R., Massa, D.L., Hutchings, J.B. 2001, in: T.R. Gull, S. Johansson \& K. Davidson (eds.), Eta Carinae and Other Mysterious Stars, ASP-CS 242, 55

King, N.L., Nota, A., Walsh, J.R., Panagia, N., Gull, T.R., Pasquali, A., Clampin, M., Bergeron, L.E. 2002, ApJ 581, 285

Morse, J.A., Kellogg, J.R., Bally, J., et al. 2001, ApJ (Letters) 548, L207

Viotti, R., Rossi, L., Cassatella, A., et al. 1989, ApJS 71, 983

Weis, K., Duschl, W.J., Chu, Y.-H. 1999, A\&A 349, 547 